\title{
Tricuspidization preferred for unicuspid aortic valve repair
}

Published online: 22 October 2012

(C) Springer Healthcare 2012

medwireNews: Unicuspid aortic valves may be reconstructed with tricuspidization using glutaraldehyde-treated autologous pericardium, with excellent early results and no mortality or major morbidity, the results of a preliminary study suggest.

Shigeyuki Ozaki and colleagues from Toho University Ohashi Medical Center, in Tokyo, Japan, note: "Some repair techniques for unicuspid aortic valve that include bicuspidization have been reported. We believe tricuspidization, rather than bicuspidization, is the ideal reconstructive technique."

They explain: "Tricuspidization can make the total length of the free margin of the aortic valve leaflets longer than is possible with bicuspidization. This appropriate length of the free margin of leaflets is necessary for the full opening of the aortic valve, which is essential for the treatment of aortic stenosis and is also necessary for the treatment of aortic regurgitation.”

Among 304 cases of aortic valve reconstruction using glutaraldehyde-treated autologous pericardium performed by the team between 2007 and 2011, nine patients with unicommissural unicuspid aortic valve were treated. Two patients had aortic stenosis, one had aortic regurgitation, and six patients had both.

Over a mean follow-up of 551 days, no reoperation or additional intervention was recorded, and all patients had a New York Heart Association functional class I classification. Without anticoagulation, no thromboembolic event was seen postoperatively.

One week postoperatively, echocardiography indicated that six had no aortic regurgitation, while three had trivial regurgitation. The average peak pressure gradient through the newly created aortic valve was $10.6 \mathrm{mmHg}$, while the average aortic valve orifice area was $2.63 \mathrm{~cm}^{2}$. Both of these measures were significant improvements over baseline.

At 1 year, echocardiography revealed that four patients had no aortic regurgitation, and five patients had trivial aortic regurgitation. The average peak pressure gradient at this follow-up was $8.6 \mathrm{mmHg}$, the team reports in the Annals of Thoracic Surgery.

By Liam Davenport, medwireNews Reporter

\section{Reference}

Ann Thorac Surg 2012; 94: 1180-1184 